\title{
Maximum Admission Capacity Model of Distribution Generation in Active Distribution Network
}

\author{
Wenjun Cao \\ North China Electric Power University Baoding, Baoding 071000, China \\ 916827591@qq.com
}

Keywords: distributed generation, active distribution network, maximum capacity.

\begin{abstract}
The optimal planning of distributed generation which intends to maximize the capacity genetration of distributed generations (DG) is mainly studied in this paper. Firstly, the probabilistic models of wind power generation and photovoltaic power generation are established under the premise of considering the uncertainties of DGs. Secondly, on the basis of this, the DG maximum admission capacity model with the maximum capacity of the DGs as the objective function is established. The model introduces different types of constraints, including voltage level constraint, line power constraint for the traditional distribution network. In addition, in order to adapt to the active management mode, we introduce DG output constraint, OLTC tap constraint, and reactive power compensation constraint.
\end{abstract}

\section{Introduction}

Energy consumption is increasing rapidly as well as the demand for electricity. At the same time, environmental problems caused by the traditional power supply are more and more serious. With the energy crisis and environmental pollution issues becoming increasingly prominent, making full use of renewable energy power generation has become a worldwide consensus.

Distributed generation (DG) refers to the power generation facility placed on the network or near the loads, using wind energy, solar energy, marine energy and other new types energy for power generation. [1]

Compared with traditional thermal power generation, DG has the advantages of small investment, clean environment protection, reliable power supply and flexible generation mode. [2] If configured properly, DG can effectively reduce the power loss, improve voltage quality and reduce the pollution of the environment. However, if the capacity of DG is unreasonable, or the configuration of multiple types of DG is not proper, it may result that the loss of the system increases, the voltage stability is reduced, thus affecting the safe production and operation of distribution network. [3]

In addition, with the large-scale DG connected to the network, the intermittency and volatility of the renewable energy will have a negative impact on the voltage, frequency and reliability of the power system, which adds difficulty to the planning and operation of the power system.

\section{DG output probability model}

Renewable energy power generation mainly refers to wind power generation, photovoltaic power generation and other unconventional energy power generation system. Renewable energy DG has the characteristics of clean, green, large reserves and wide distribution. However, since the energy of wind power comes from the wind energy of nature, and the wind speed has a strong randomness of volatility, so the wind power output has a large uncertainty. Photovoltaic power generation is also affected by external factors such as light intensity and has a random characteristic. The uncertainties of renewable energy DG have brought unprecedented challenges to the planning and operation of power systems. Therefore, it is necessary to consider its uncertainty when the optimizing the configuration of DG. [4] 


\subsection{Wind power generation probability model.}

In mathematical statistics, the stochastic properties of a physical quantity are usually described by a probability distribution model. Therefore, we can obtain a probability distribution model for obtaining wind speed in a given area and obtain probabilistic model for obtaining wind power output according to the relationship between wind power output power and wind speed. In general, wind speed distribution is subject to Weibull distribution and the probability density function is:

$$
f(v)=\frac{k_{w}}{C_{w}}\left(\frac{v}{C_{w}}\right)^{k_{w}-1} \exp \left[-\left(\frac{v}{C_{w}}\right)^{k_{w}}\right]
$$

The relationship between the wind power output and the wind speed can be expressed as a piecewise function:

$$
P_{W T}=\left\{\begin{array}{c}
0, \quad v \leq v_{c i}, v \geq v_{c o} \\
P_{W T_{-} n} \frac{v-v_{c i}}{v_{n}-v_{c i}}, v_{c i} \leq v \leq v_{n} \\
P_{W T_{-} n}, v_{n} \leq v \leq v_{c o}
\end{array}\right.
$$

where $k_{w}$ and $c_{w}$ are the shape index and scale index of Weibull distribution, $P_{W T_{-} n}$ is the rated power of wind power, $v, v_{n}, v_{c i}$ and $v_{c o}$ are the actual wind speed, rated wind speed, the cut-in wind speed and the cut-out wind speed.

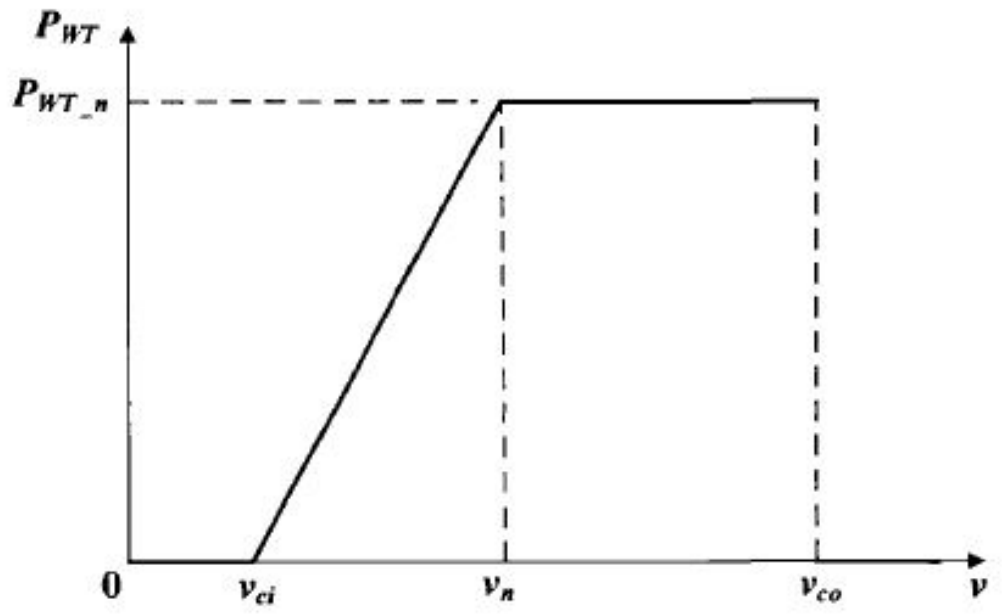

Fig.1 Relationship between wind turbine generators' output power and wind speed

\subsection{Photovoltaic power generation probability model.}

Photovoltaic power is mainly determined by the intensity of sunlight. According to statistics, the intensity of solar light is similar to the Beta distribution in a certain period of time. The probability density function can be expressed by the following formula:

$$
f(r)=\frac{\Gamma(\propto+\beta)}{\Gamma(\propto) \Gamma(\beta)}\left(\frac{r(t)}{r_{\max }}\right)^{\propto-1}\left(1-\frac{r(t)}{r_{\max }}\right)^{\beta-1}
$$

where: $r_{\max }$ is the maximum intensity of light in a certain period of time; $\propto, \beta$ are respectively Beta distribution shape parameter.

The relationship between the output power of the photovoltaic power generation and the intensity of light is:

$$
P_{s}(t)=P_{\text {stc }} \frac{r(t)}{r_{\text {stc }}}\left[1+k\left(T(t)-T_{\text {stc }}\right)\right]
$$

where, $P_{s}(t)$ is the output power of the photovoltaic cell when the intensity of light is t; $T(t)$ is the surface temperature of the PV array. $r_{\text {stc }}, T_{\text {stc }}, P_{\text {stc }}$ are the light temperature, the temperature of the 
PV array and the maximum output power under the standard test environment with $1 \mathrm{~kW} / \mathrm{m}^{2}$, $25{ }^{\circ} \mathrm{C}$. $\mathrm{K}$ is the temperature coefficient.

\section{DG maximum accession capacity model}

\subsection{Objective function}

The objective function used to describe the maximum admission capacity of DG is:

$$
\max \sum_{i=1}^{n} P_{D G i}
$$

where $n$ is the selected DG access node, $P_{D G i}$ is the active power of DG for the i-th node.

\subsection{Constraints}

Constraints of traditional distribution networks include power flow constraint, voltage level constraint and line power constraint.

- Power flow constraint

$$
\begin{aligned}
& P_{G i}-P_{L i}=U_{i} \sum_{k \in A(i)} U_{k}\left(G_{I K} \cos \delta_{i k}+B_{i k} \sin \delta_{i k}\right) \\
& Q_{G i}-Q_{L i}=U_{i} \sum_{k \in A(i)} U_{k}\left(G_{I K} \sin \delta_{i k}-B_{i k} \cos \delta_{i k}\right)
\end{aligned}
$$

$P_{G i}, Q_{G i}$ are active and reactive power output of power supply at node $i . P_{L i}, Q_{L i}$ are the active and reactive loads at node $i . U_{i}, U_{k}$ are voltage amplitude of node $i$ and node $k . \delta_{i k}$ is phase difference of node voltage. $G_{I K}$ and $B_{i k}$ are system admittance.

- Voltage level constraint

$$
V_{\text {imin }} \leq V_{i} \leq V_{\text {imax }}
$$

$V_{i}, V_{\text {imin }}$ and $V_{\text {imax }}$ are the voltage, the minimum voltage and the maximum voltage allowed of node $i$.

- Line power constraint

$$
S_{j} \leq S_{\text {jmax }}
$$

$S_{j}$ is the apparent power through line $j$ and $S_{j \max }$ is line transmission capacity limit.

In order to solve a great of DGs being connected into the distribution network, improve DGs utilization, optimize the primary energy structure and network compatibility and other issues, active distribution network technology (ADN) came into being.

Now, there are three kinds of active management measures adopted in DG planning, for the three measures, we propose three constraints.

- DG output constraint

Distributed generator output control: By controlling the output of the DG in the node, the node voltage is adjusted and the network power distribution is improved.

$$
P_{\text {DGimin }} \leq P_{D G i} \leq P_{\text {DGimax }}
$$

$P_{D G i}, P_{D G i m i n}$ and $P_{D G i m a x}$ are the active power of DG, the minimum active power of DG and the maximum active power of DG of node $i$.

- OLTC tap constraint

Adjust the OLTC tap: Adjust the variable edge position of the OLTC primary side so that the voltage of the network node is controlled within the safe range.

$$
T_{k \min } \leq T_{k} \leq T_{k \max }
$$

$T_{k}, T_{k \min }$ and $T_{k \max }$ are the tap position, the minimum tap and the maximum tap of the transformer $k$.

- Reactive power compensation constraint 
Switch the reactive power compensation device: In the DG access point, through switching reactive power compensation equipment, absorb or release reactive power to change the network reactive power distribution, so that the distribution network distribution and voltage levels can be improved.

$$
Q_{\text {Cimin }} \leq Q_{C i} \leq Q_{\text {Cimax }}
$$

$Q_{C i}, Q_{C i m i n}$ and $Q_{C i m a x}$ are the reactive power output of reactive power compensation device, minimum reactive power and maximum reative power of node $i$.

\section{Summary}

The paper firstly analyzes the background and significance of the research and investigates the development status of DG and the development and research status of ADN. The characteristics of ADN and typical DG are studied. On this basis, the probability model of wind power generation is established, the DG maximum admission capacity model in ADN is established.

Based on the above discussion, this paper constructs the DG maximum admission capacity model and puts forward the optimal solving method to obtain optimal allocation scheme of DG. The research on the optimal allocation of DG considering the uncertain factors of renewable energy generation can better reflect the characteristics of large-scale DG connecting with network in the future and meet the future development trend of power network, which is important for the development of energy network and active distribution network.

From the model, three conclusions can be given:

(1) The model considering the indeterminacy of wind and solar power is more practical and can be applied in the electrical power system

(2) The active management has been in consideration, which improve the capacity of the DG.

(3) The optimization of DG includes all main constrains, which can get the maximum capacity under the reliable and safe operation of the electric power system.

\section{References}

[1]. Dugan R C, McDermott T E. Distributed generation [J]. IEEE Industry Application Magazine, 2002, 8(2), 19-25.

[2]. Guerrero J M, Blaabjerg F, Zhelev T, et al. Distributed generation: Toward a new energy paradigm[J]. IEEE Industrial Electronics Magazine, 2010, 4(1): 52-64.

[3]. Kashem M A, Le A D T, Negnevitsky M, et al. Distributed generation for minimization of power losses in distribution systems[C]//Power Engineering Society General Meeting, 2006. IEEE. IEEE, 2006: 8 pp.

[4]. Zou K, Agalgaonkar A P, Muttaqi K M, et al. Distribution system planning with incorporating DG reactive capability and system uncertainties[J]. IEEE Transactions on Sustainable Energy, 2012, 3(1): 112-123.

[5]. Clement-Nyns K, Haesen E, Driesen J. The impact of charging plug-in hybrid electric vehicles on a residential distribution grid[J]. IEEE Transactions on Power Systems, 2010, 25(1): 371-380. 